\title{
The utilization of an ocular wound chamber on corneal epithelial wounds
}

This article was published in the following Dove Press journal:

Clinical Ophthalmology

\author{
Jennifer S McDaniel' \\ Andrew W Holt' \\ Elaine D Porl \\ Elof Eriksson ${ }^{2}$ \\ Anthony J Johnson' \\ Gina L Griffith' \\ 'Department of Sensory Trauma, \\ United States Army Institute of \\ Surgical Research, San Antonio, \\ TX, USA; ${ }^{2}$ Harvard Medical School, \\ Boston, MA, USA
}

Correspondence: Gina L Griffith Department of Sensory Trauma, United States Army Institute of Surgical Research, 3698 Chambers Pass, JBSA Fort Sam Houston, TX 78234-7767, USA $\mathrm{Tel}+\mathrm{I} 2105398585$

$\mathrm{Fax}+\mathrm{I} 2105396266$

Email gina.l.griffith8.mil@mail.mil

\begin{abstract}
Purpose: Currently available ocular moisture chambers are not adequate to manage the treatment of periocular burns, corneal injuries, and infection. The purpose of these studies was to demonstrate that a flexible, semi-transparent ocular wound chamber device adapted from technology currently used on dermal wounds is safe for use on corneal epithelial injuries.
\end{abstract}

Materials and methods: A depilatory cream (Nair ${ }^{\mathrm{TM}}, 30$ seconds) was utilized to remove the excess hair surrounding the left eyes of anesthetized Institute Armand Frappier (IAF) hairless, female guinea pigs (Crl:HA-Hrhr). A $4 \mathrm{~mm}$ corneal epithelium defect was created using a corneal rust ring remover (Algerbrush ${ }^{\circledR} \mathrm{II}$ ). Epithelial defects were either left untreated or the eyes were fitted with an ocular wound chamber and $0.5 \mathrm{~mL}$ of hydroxypropyl methylcellulose (HPMC) gel $\left(\mathrm{GenTeal}^{\circledR}\right)$ or HPMC liquid $\left(\mathrm{GenTeal}^{\circledR}\right)$ was injected into each chamber $(\mathrm{N}=5$ per group). At $0,24,48$, and 72 hours fluorescein and optical coherence tomography imaging was collected and the intraocular pressure (IOP) was measured. H\&E staining was performed on corneal and eyelid skin samples and evaluated by a veterinary pathologist.

Results: Corneal epithelial wounds demonstrated $100 \%$ closure rates when left untreated or treated with an ocular wound chamber containing HPMC gel at 72 hours while wounds treated with an ocular wound chamber containing HPMC liquid were 98\% healed. No significant differences were found in corneal thickness and wound healing, IOP, or eyelid skin pathology in any treatment group when compared to controls.

Conclusions: This study indicates that adapted wound chamber technology can be safely used on sterile, corneal epithelial wounds without adverse effects on periocular or ocular tissue when filled with a liquid or gel.

Keywords: corneal epithelial wound healing, periocular burns, wound chamber, exposure keratopathy, guinea pig

\section{Introduction}

Between $7.5 \%$ and $27 \%$ of patients with facial burns experience some ocular trauma secondary to the initial burn injury such as exposure keratopathy or subsequent infection. ${ }^{1,2}$ Exposure keratopathy, which has been observed in up to $60 \%$ of ICU patients, ${ }^{3,4}$ occurs when the ocular surface is exposed to the environment for an extended length of time such as when the eyelids are damaged and unable to close or provide adequate ocular coverage. This exposure may subsequently lead to corneal epithelial damage or PCEDs, defects that fail to heal over a 2 -week period. ${ }^{5}$ If PCEDs are not effectively treated, corneal ulceration, scarring, and, in more severe cases, partial or complete vision loss can occur. ${ }^{6}$ In the presence of even minor defects, intensive treatment including the administration of hourly drops should be initiated. ${ }^{2}$ As ophthalmic drops rapidly dissipate after administration, therapies that involve the frequent administration of drops require a high burden of care and patient compliance. 
Other therapeutic options for patients suffering from exposure keratopathy include bandage or scleral contact lenses ${ }^{7}$ and the use of more invasive surgical interventions which are amniotic membrane grafting ${ }^{7,8}$ or tarsorrhaphy. ${ }^{7}$ Current therapies tend to be ineffective thereby prolonging healing time and a patient's impaired visual acuity. ${ }^{7}$ Due to the challenges encountered in treating these patients, clinicians require more effective treatment modalities for the management of persistent corneal epithelial defects.

The cornea is comprised of five well-characterized layers from the anterior to the posterior region, respectively: epithelium, Bowman's layer, stroma, Descement's membrane, and endothelium. ${ }^{9}$ As the outermost layer of the cornea, the epithelium provides an important protective barrier to the rest of the eye against noxious environmental stimuli and inflammatory mediators in the tear film. Furthermore, a smooth epithelial surface is essential for proper light refraction and consequently, proper vision. ${ }^{10}$ If the corneal epithelium is compromised, not only is visual acuity impacted, but the cornea is also susceptible to infection. ${ }^{11}$ Therefore, increasing the rate of re-epithelialization can decrease the likelihood that patients will develop an ocular infection or other complications such as corneal melting. To this end, ocular therapies often seek to enhance corneal epithelial wound closure by modulating cellular proliferation, migration, and adhesion. ${ }^{13}$ As a result of the need for therapies to treat these defects and provide protection to the eye, we have begun exploring therapies to address the clinical challenges associated with treating patients suffering from epithelial defects and/or keratitis infections secondary to corneal trauma. We aim to explore novel ophthalmic treatment modalities to meet the needs of both clinicians and patients.

In this study, we investigated the use of an OWC device adapted from a PWD currently used in dermal burn patients to protect the eye and facilitate corneal wound closure. ${ }^{14-16}$ Comprised of a flexible adhesive base that attaches to the perimeter of the eye and enclosed by a transparent cover, the OWC provides protection from the external environment while allowing for visual monitoring of injured tissues (Figure 1). The PWD supports a fluid-filled environment to protect healing skin wounds while consistently delivering precise volumes and concentrations of therapeutics such as antibiotics. Previous studies have shown that the utilization of the PWD to deliver concentrated amounts of antibiotics to infected dermal wounds resulted in decreased bacterial loads and a significant reduction in inflammatory mediators. ${ }^{17,18}$ As a result of the success of the PWD on dermal wounds, OWC technology was adapted to provide a novel treatment for facial burn patients while providing ocular protection and promoting healing and re-epithelialization of the ocular surface. Analyses of multiple randomized, controlled trials have demonstrated the use of commercially available ocular moisture chambers in conjunction with lubricating ointments such as mineral oil and petrolatum-based ointments are more effective at preventing exposure keratopathy than lubricants and eyelid taping alone. ${ }^{3,4}$ Currently available moisture chambers, however, do not hold sufficient volumes of fluid to cover the eye and tissues as they neither provide a watertight seal nor are they capable of delivering precise and continuous doses of therapeutics to the injured tissue. As a result, we hypothesized that our adapted OWC device would provide enough fluid to cover the eye and periocular tissues allowing for a more advanced treatment for exposure keratopathy, PCEDs, and periocular burns while protecting the periorbital tissue and ocular surface in cases of facial burns that result in insufficient eyelid coverage. If successful, the adapted OWC would decrease the risk of
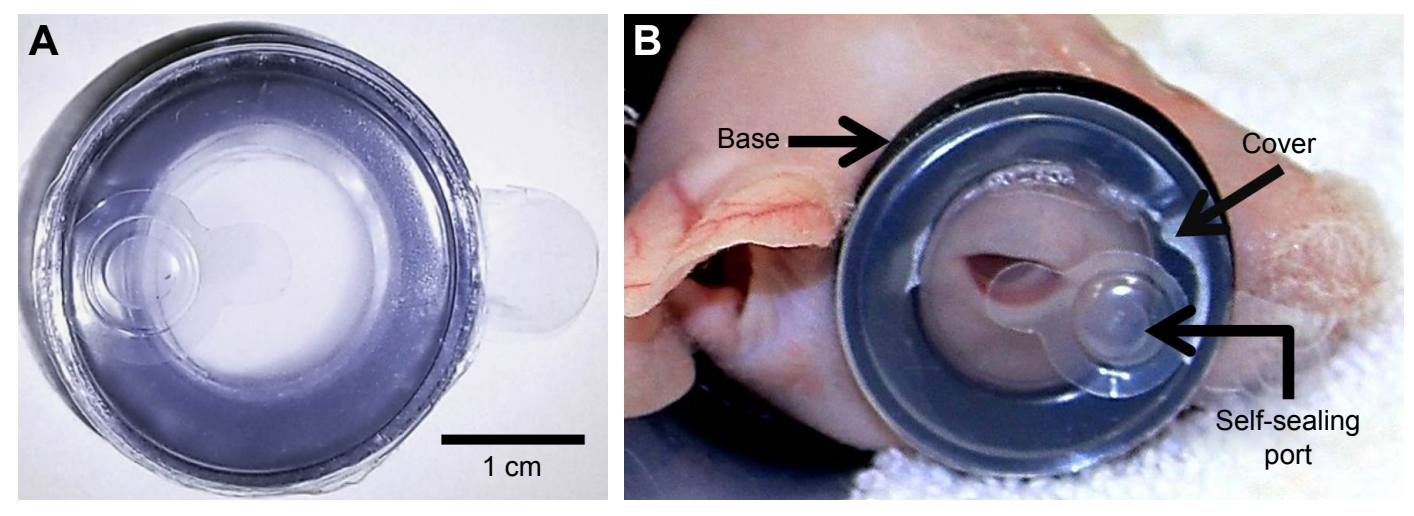

Figure I An OWC measuring $3 \mathrm{~cm}$ in diameter has been developed for ocular use with a flexible base that attaches to the perimeter of the eye (A). The device has a transparent cover and a self-sealing port that allows for the delivery of therapeutics (A). The OWC has been specifically designed for use in our guinea pig model (B) and provides a watertight seal that allows for the creation of a controlled microenvironment over the eye. The base, cover, and self-sealing port are labeled accordingly as indicated by arrows (B).

Abbreviation: OWC, ocular wound chamber. 
ocular infection and exposure keratopathy while allowing periocular tissues time to heal accordingly.

\section{Material and methods Animals}

Female IAF hairless, guinea pigs (200-250 g; Crl:HA-Hr ${ }^{\text {hr }}$ ) were obtained from Charles River Laboratories (Wilmington, MA, USA). Guinea pigs were acclimated 72 hours prior to experimentation and procedures performed on anesthetized guinea pigs. Research was conducted in compliance with the Animal Welfare Act, the implementing Animal Welfare Regulations, and the principles of the Guide for the Care and Use of Laboratory Animals, National Research Council. The facility's IACUC approved all research conducted in this study. The facility where this research was conducted is fully accredited by Association for Assessment and Accreditation of Laboratory Animal Care International.

\section{In vivo corneal wound creation}

Prior to the creation of the corneal epithelial defect, isoflurane titrated to $5 \%$ with oxygen was administered for $2 \mathrm{~min}$ before the administration of ketamine (Ketacine ${ }^{\mathrm{TM}} \mathrm{HCl}$, 20-40 mg/kg; VetOne ${ }^{\circledR}$, Boise, ID, USA) and dexmedetomidine hydrochloride $\left(0.5 \mathrm{mg} / \mathrm{kg}\right.$; Putney ${ }^{\circledR}$, Overland Park, KS, USA). The corneal epithelium was removed from the left eyes of anesthetized guinea pigs using a corneal rust ring remover (Algerbrush ${ }^{\circledR} \mathrm{II}$; Alger Company Inc., Lago Vista, TX, USA) as previously described while right eyes were uninjured and served as controls. ${ }^{19}$ Briefly, corneal epithelial defects were created centrally by demarcating the cornea using a $4 \mathrm{~mm}$, disposable biopsy punch (Miltex, York, PA, USA) and removing the epithelium with a corneal rust ring remover equipped with a $1 \mathrm{~mm}$ rotating burr. The corneal epithelial defects were rinsed with sterile BSS (Alcon, Fort Worth, TX, USA) and stained with fluorescein utilizing fluorescein sodium ophthalmic strips (Fluorets; Laboratoire Chauvin, Aubenas, France), dampened with $100 \mu \mathrm{L}$ sterile BSS. Uptake of fluorescein staining was visualized using a surgical microscope equipped with a cobalt blue filter and camera (Lumera 700; Carl Zeiss Surgical, Oberkochen, Germany). Images were obtained at $0,24,48$, and 72 hours post injury immediately following staining with fluorescein. The area of each corneal epithelial defect was measured and quantitated using ImageJ software (U.S. National Institutes of Health, Bethesda, MD, USA). The area of each wound was expressed as a percentage of the original wound size. The results are reported as percent wound closure and mean \pm SEM of five guinea pigs per group. Upon completion of all procedures, anesthesia was reversed using atipamezole hydrochloride $(0.5 \mathrm{mg} / \mathrm{kg}$; Orion Pharma, Espoo, Finland) at a dose equal to the dexmedetomidine administered.

\section{Ocular wound chamber placement}

Before corneal epithelial defect creation and chamber placement, the left eyes of guinea pigs were protected with a petrolatum and mineral oil ointment (Larci-Lube ${ }^{\circledR}$; Allergan, Parsippany-Troy Hills, NJ, USA) and excess hair removed with a depilatory cream (Nair ${ }^{\mathrm{TM}}, 30$ seconds). The skin was rinsed with BSS and cleaned with $70 \%$ ethanol. Animals were fitted with the OWC (Applied Tissue Technologies, Hingham, MA, USA) (Figure 1A) over the wounded left eye of the guinea pig (Figure 1B). Guinea pigs were randomly grouped ( $\mathrm{N}=5$ per group) and $0.5 \mathrm{~mL}$ of HPMC liquid (GenTeal Lubricant Eye Drops Moderate Dry Eye Relief; Novartis Pharmaceuticals Corporation, East Hanover, NJ, USA) or HPMC gel (GenTeal Lubricant Eye Gel Severe Dry Eye Relief; Novartis Pharmaceuticals Corporation, East Hanover, NJ, USA) was injected into the chamber through a self-sealing attached port using a 25 gauge 1 " needle. One group of injured animals received no chamber or additional treatment. The chambers were replaced daily and filled with fresh HPMC liquid or HPMC gel. Both injured and uninjured eyes were assessed daily at $0,24,48$, and 72 hour post chamber placement with tonometry for IOP, OCT for corneal thickness, white light, and fluorescein imaging for epithelial defects.

\section{Rebound tonometry}

A tonometer (Icare ${ }^{\circledR}$; TonoLab, Finland) was used to take an average of five independent IOP measurements at 0,24, 48, and 72 hours while animals were under anesthesia. Measurements were taken pre- and postwound creation. Results are presented as mean \pm SEM of five guinea pigs per group.

\section{Optical coherence tomography}

Spectral Domain Optical Coherence Tomography (SD-OCT) (Micron IV; Phoenix Research Laboratories, Pleasanton, CA, USA) corneal images of wounded and control eyes were obtained via integrated image acquisition software (Phoenix Research Laboratories) for each animal at 0, 24, 48, and 72 hours. Central corneal thickness was measured using ImageJ analysis software (NIH, Bethesda, MD, USA). Results are presented as mean \pm SEM of five guinea pigs per group. 


\section{Histology}

Following humane euthanasia, whole eye globes and skin samples from both wounded and control animals were collected for histology at 72 hours. Whole globes were placed in modified Davidson's solution (Poly Scientific R\&D Corporation, Bay Shore, NY, USA) immediately following tissue collection for 24 hours for the initial fixation and then transferred to $10 \%$ NBF (Thermo Fisher Scientific, Middletown, VA, USA). Vertically bisected globes were placed (cut side down) in a megatissue cassette (Fisher Scientific, Fair Lawn, NJ, USA). Upper and lower eyelids skin samples (including sections of skin contacted with the wound chamber adhesive) were placed in 10\% NBF. Eye and skin samples were processed, embedded in paraffin, sectioned at $4 \mu \mathrm{m}$ and stained with H\&E (Fisher Scientific, Fair Lawn, NJ, USA) according to manufacturer's directions. Histological sections were scored by a board certified veterinary pathologist using a scoring system created in-house as described in Table 1. Gradations of neovascularization, keratitis, and uveitis/conjunctivitis were determined for cornea samples while dermal inflammation, epithelial hyperplasia, and the presence or absence of pustules and folliculitis were identified in skin sections. Total number of epithelial mitotic figures were counted from limbus to limbus on cornea sections.

\section{Statistical analysis}

Statistics were calculated and graphed using GraphPad Prism 7 (GraphPad Software, Inc., La Jolla, CA, USA). Wound healing, corneal thickness, and IOP data were analyzed

Table I Pathology scoring system

\begin{tabular}{|c|c|c|}
\hline \multicolumn{3}{|l|}{ Cornea } \\
\hline $\begin{array}{l}\text { Neovascularization/ } \\
\text { uveitis/conjunctivitis }\end{array}$ & Keratitis & $\begin{array}{l}\text { Epithelial } \\
\text { mitotic figures }\end{array}$ \\
\hline $\begin{array}{l}0-\text { Absent } \\
I-\text { Present }\end{array}$ & $\begin{array}{l}0 \text { - None } \\
\text { I - Minimal } \\
2 \text { - Mild } \\
3 \text { - Moderate } \\
4 \text { - Marked }\end{array}$ & $\begin{array}{l}\text { Number of } \\
\text { mitotic figures } \\
\text { counted limbus } \\
\text { to limbus }\end{array}$ \\
\hline \multicolumn{3}{|l|}{ Skin } \\
\hline $\begin{array}{l}\text { Dermal } \\
\text { inflammation }\end{array}$ & $\begin{array}{l}\text { Epithelial } \\
\text { hyperplasia }\end{array}$ & $\begin{array}{l}\text { Pustules/ } \\
\text { folliculitis }\end{array}$ \\
\hline 0 - None & 0 -None & 0 - Absent \\
\hline I - Minimal & $\begin{array}{l}\text { I - Mild }(2 \times \text { normal } \\
\text { thickness) }\end{array}$ & I - Present \\
\hline 2 - Mild & $\begin{array}{c}2 \text { - Moderate }(3-5 \times \\
\text { normal thickness) }\end{array}$ & \\
\hline 3 - Moderate & $\begin{array}{l}3 \text { - Marked (>5× } \\
\text { normal thickness) }\end{array}$ & \\
\hline 4 - Marked & & \\
\hline
\end{tabular}

using two-way ANOVA followed by a Bonferroni's multiple comparisons test. Pathology scores were analyzed using the nonparametric Kruskal-Wallis test. Independent values are represented as the mean \pm SEM. A $P$-value of $<0.05$ was regarded as significant for all statistical analyses.

\section{Results}

\section{Corneal epithelial wound healing}

To investigate the ability of corneal epithelial defects to heal in a fluid filled environment, corneal epithelial wounds were created, left uncovered or covered with an ocular wound chamber and filled with either HPMC gel or HPMC liquid. The wounds were monitored by white light imaging to visually observe any areas of corneal opacity (Figure 2A) while epithelial closure was monitored via fluorescein uptake for all animal groups (Figure 2B). Untreated corneal epithelial wounds were $83 \%$ re-epithelialized at 24 hours while corneal wounds treated with the ocular wound chamber filled with HPMC gel were $81 \%$ re-epithelialized and those treated with HPMC liquid were $71 \%$ re-epithelialized (Figure 2C). At 48 hours post wound creation, wounds were $99 \%, 100 \%$, and $87 \%$ re-epithelialized when left untreated or treated with an OWC in conjunction with HPMC gel or HPMC liquid respectively. By 72 hours postwounding, nontreated injuries and HPMC gel were found to be $100 \%$ re-epithelialized while HPMC liquid treated wounds were shown to be $98 \%$ re-epithelialized.

\section{Corneal thickness}

To assess the response of injured corneas to prolonged liquid exposure and monitor changes in corneal thickness, OCT was employed. At each time point, OCT images were collected on injured eyes and the corneal thickness was measured (Figure 3A). Immediately after injury, no differences in corneal thickness were observed among groups indicating consistency in injury creation (Figure 3B). By 24 hour postinjury, all injured groups exhibited significant $(P<0.05)$ increases in corneal thickening compared to the uninjured controls (Figure 3B). The HPMC liquid group showed continued corneal swelling at 48 hours compared to all other groups, while the no chamber and HPMC gel groups were stabilized and at 72 hours, they began to decrease.

\section{IOP}

IOP was measured to detect changes in pressure as a result of injury, chamber placement, and therapeutic application. Preinjury, combined guinea pigs averaged an IOP of $10.19 \pm 0.43 \mathrm{mmHg}$ (mean \pm SEM). Immediate postinjury IOP 
A
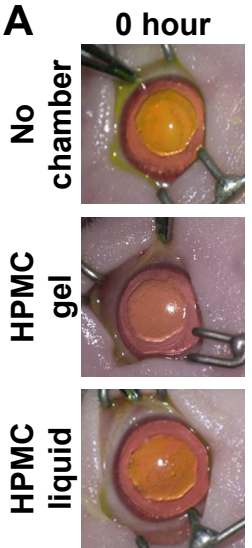

24 hours
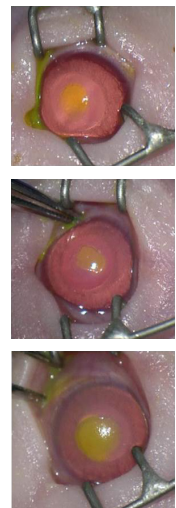
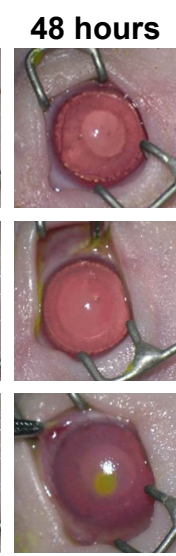

72 hours

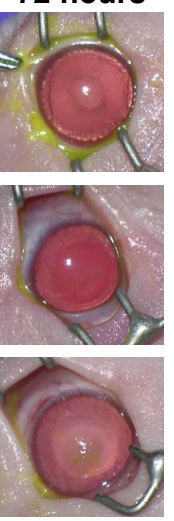

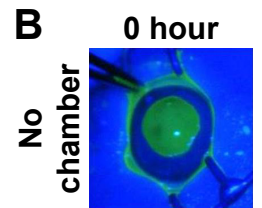

24 hours
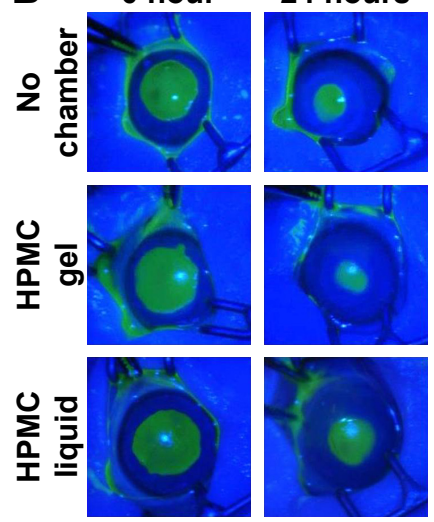

48 hours
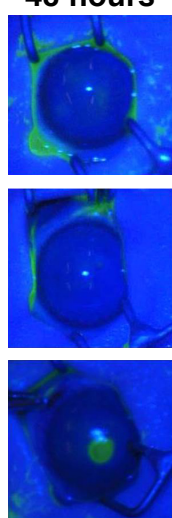

C

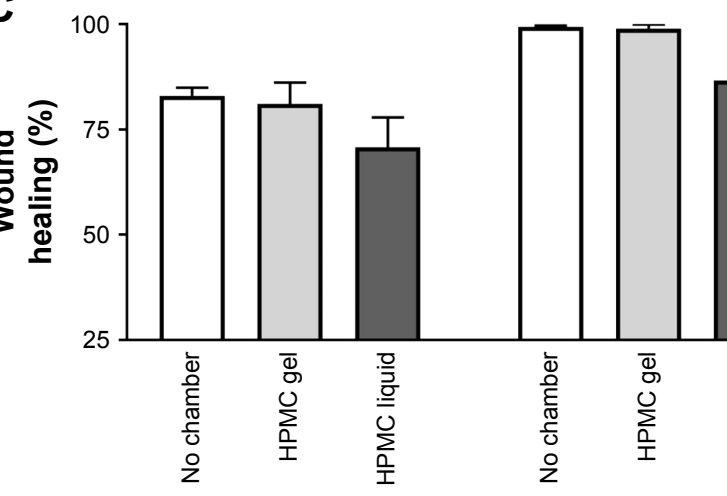

24 hours

48 hours

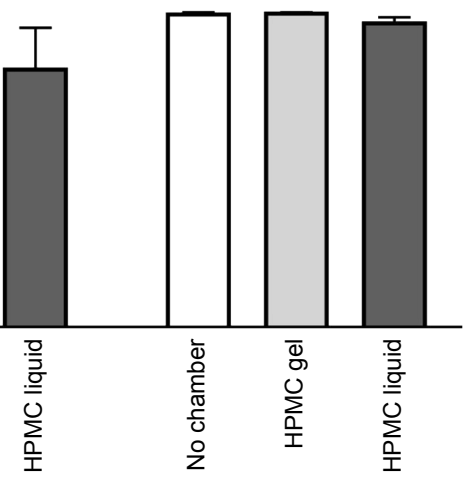

72 hours

72 hours
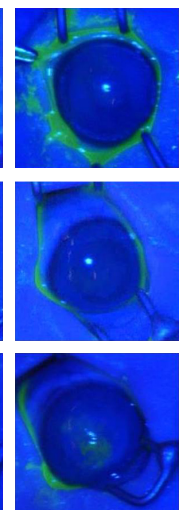

Figure 2 Re-epithelialization of corneal wounds. Representative images of white light (A) and fluorescein stained (B) injured eyes at 0 , 24, 48, and 72 hours. Comparison of percentage wound closure between groups over time $(\mathbf{C})$. No statistically significant differences across treatment groups. $\mathrm{N}=5$ animals per group.

Abbreviation: HPMC, hydroxypropyl methylcellulose.

measurements revealed an average IOP of $11.20 \pm 1.07 \mathrm{mmHg}$ in uninjured control animals, and $10.93 \pm 0.55 \mathrm{mmHg}$ in the injured groups. By 72 hours postwounding, the IOP of treatment animals containing either HPMC gel or liquid averaged $10.20 \pm 0.20 \mathrm{mmHg}$ and $10.40 \pm 1.03 \mathrm{mmHg}$, respectively (Figure 4). Overall, no significant changes in IOP measurements were observed between the groups tested over time or as a result of injury or applied therapeutics.

\section{Eye histology}

To histologically evaluate the effects of corneal injury creation, chamber placement, and HPMC gel and liquid application on the eyes, whole globes were collected at the time of humane euthanasia and submitted for pathology. H\&E stained corneal sections (Figure 5A) were scored by a blinded veterinary pathologist utilizing a scoring matrix. Each sample received a score as described in Table 1 (Figure 5B). Evidence of neovascularization, keratitis, and uveitis/conjunctivitis was observed in corneal sections of most injured groups; however, the clinical significance was negligible. The presence of epithelial mitotic figures in injured cornea sections indicated increased cellular proliferation and re-epithelialization and was not significantly different among injured groups (Figure 5C).

\section{Skin histology}

Skin eyelid histological samples were assessed by a veterinary pathologist to identify inflammation and epithelial hyperplasia due to chamber placement and therapeutic application (Figure 6A). The HPMC gel group showed a significant increase in dermal inflammation as indicated by the infiltration of heterophils $(P<0.02)$ compared to the no chamber group and a significant increase in epithelial hyperplasia $(P<0.005)$ compared to no chamber and HPMC liquid animal groups (Figure 6B). No significant differences were observed in the histological sections in pustule formation or folliculitis across the groups.

\section{Discussion}

As current therapeutic options for periocular burns, corneal injuries, and subsequent infection are limited, improved and effective treatments and/or technologies are needed for the 

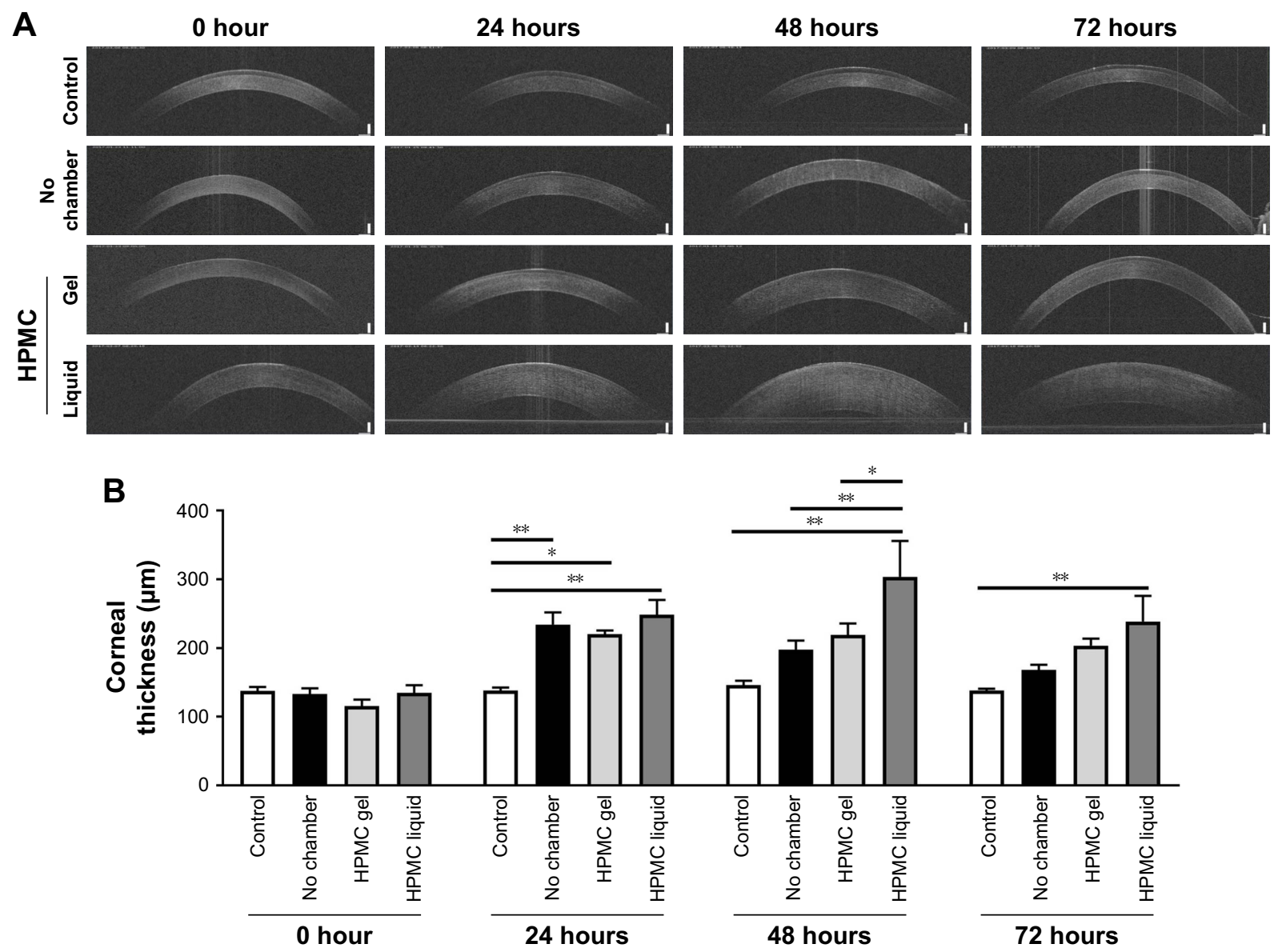

Figure 3 Corneal thickness. OCT of control, no chamber, HPMC gel- and liquid-treated guinea pig corneas at 0, 24, 48, and 72 hours (A). Comparison of central corneal thickness between groups over time (B). $\mathrm{N}=5$ animals per group. $* P<0.05 ; * * P<0.005$.

Abbreviations: HPMC, hydroxypropyl methylcellulose; OCT, optical coherence tomography.

management of these injuries. One therapy that has been utilized to treat these types of injuries is the moisture chamber. ${ }^{20}$ Moisture chambers are designed to increase the humidity of the ocular surface to treat ocular conditions such as dry eye syndrome, exposure keratopathy, and lagophthalmos. ${ }^{20,21}$ While moisture chambers increase the humidity surrounding injured tissues, unlike the OWC, moisture chambers do not provide a watertight seal. This watertight seal is a critical

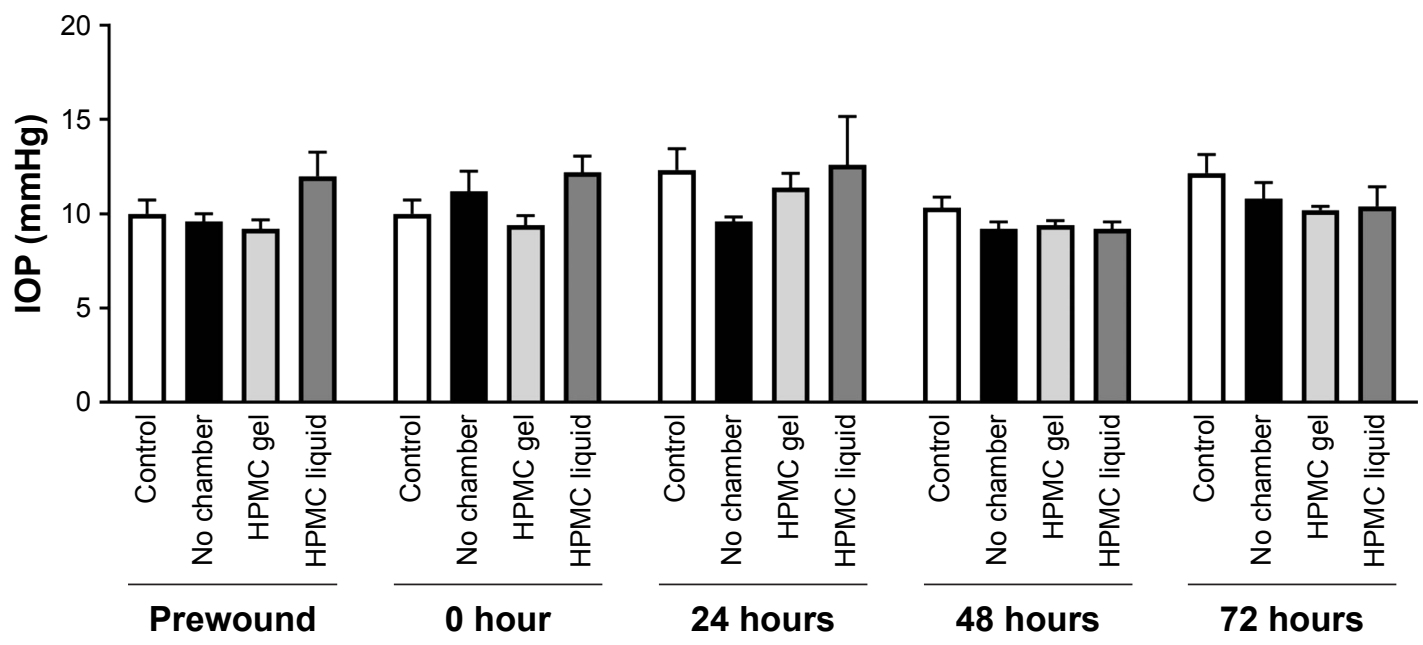

Figure 4 IOP of treated and untreated guinea pig eyes at $0,24,48$, and 72 hours. No statistically significant differences across treatment groups. $\mathrm{N}=5$ animals per group. Abbreviations: IOP, intraocular pressure; HPMC, hydroxypropyl methylcellulose. 
A

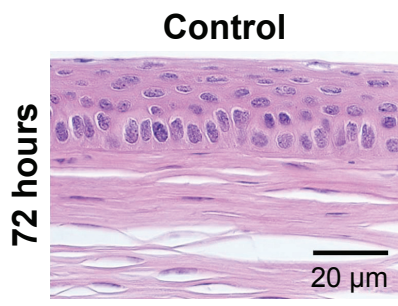

No chamber

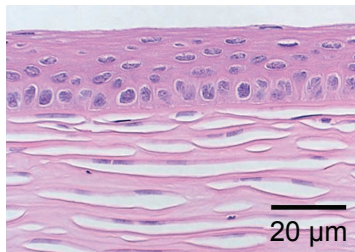

HPMC gel

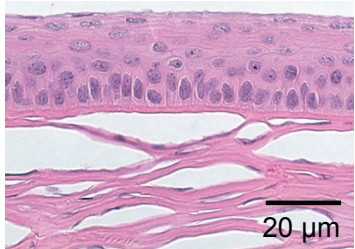

HPMC liquid

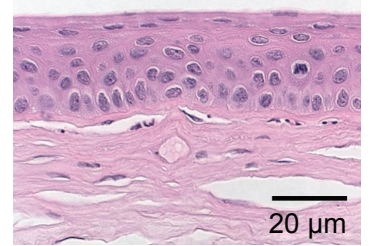

B

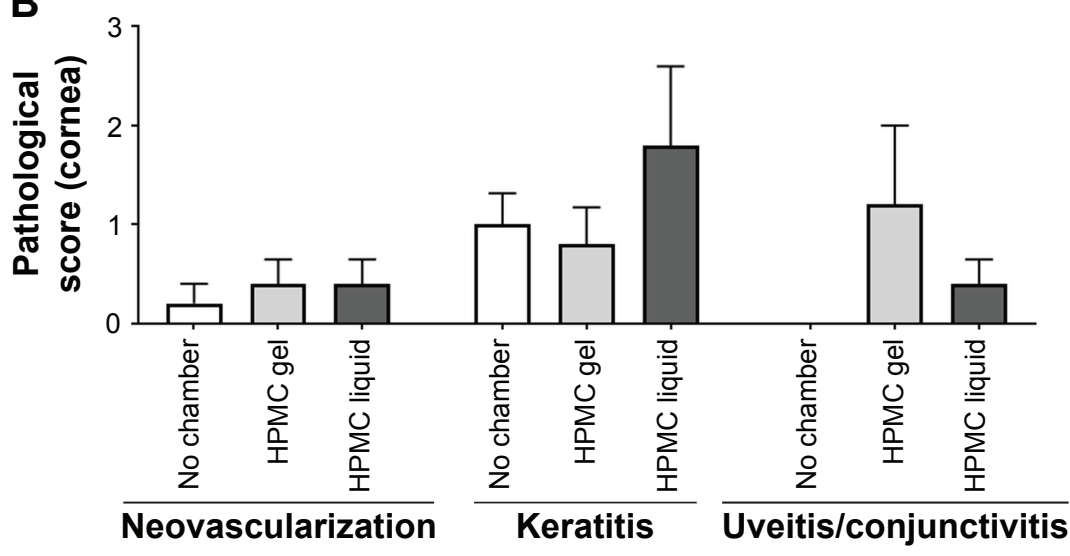

C

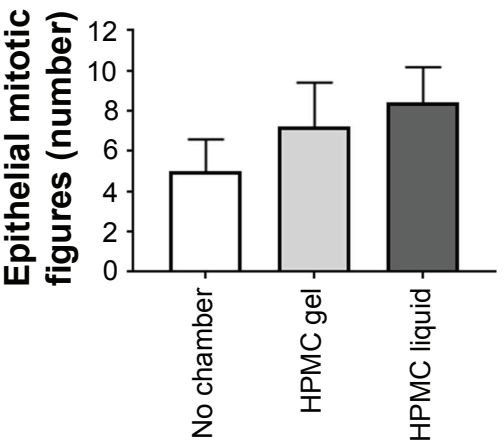

Figure 5 Histological analysis of guinea pig corneas. Representative images of hematoxylin and eosin stained cornea sections (A). Comparison of key pathology observations between treatment groups (B). Comparison of total number of epithelial mitotic figures per group (C). No clinically relevant findings or any significant differences were observed. Abbreviation: HPMC, hydroxypropyl methylcellulose.

A

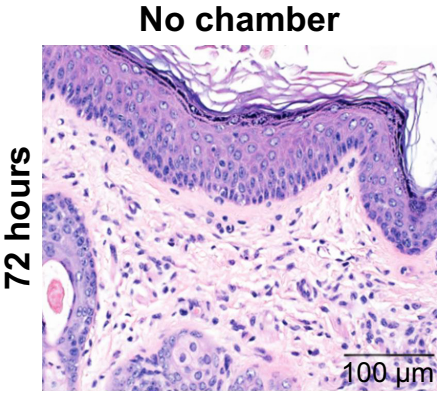

HPMC gel

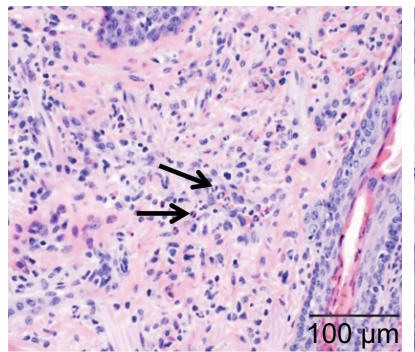

HPMC liquid

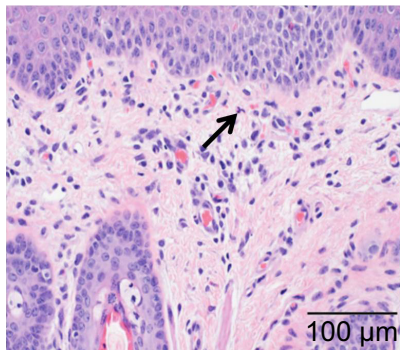

B

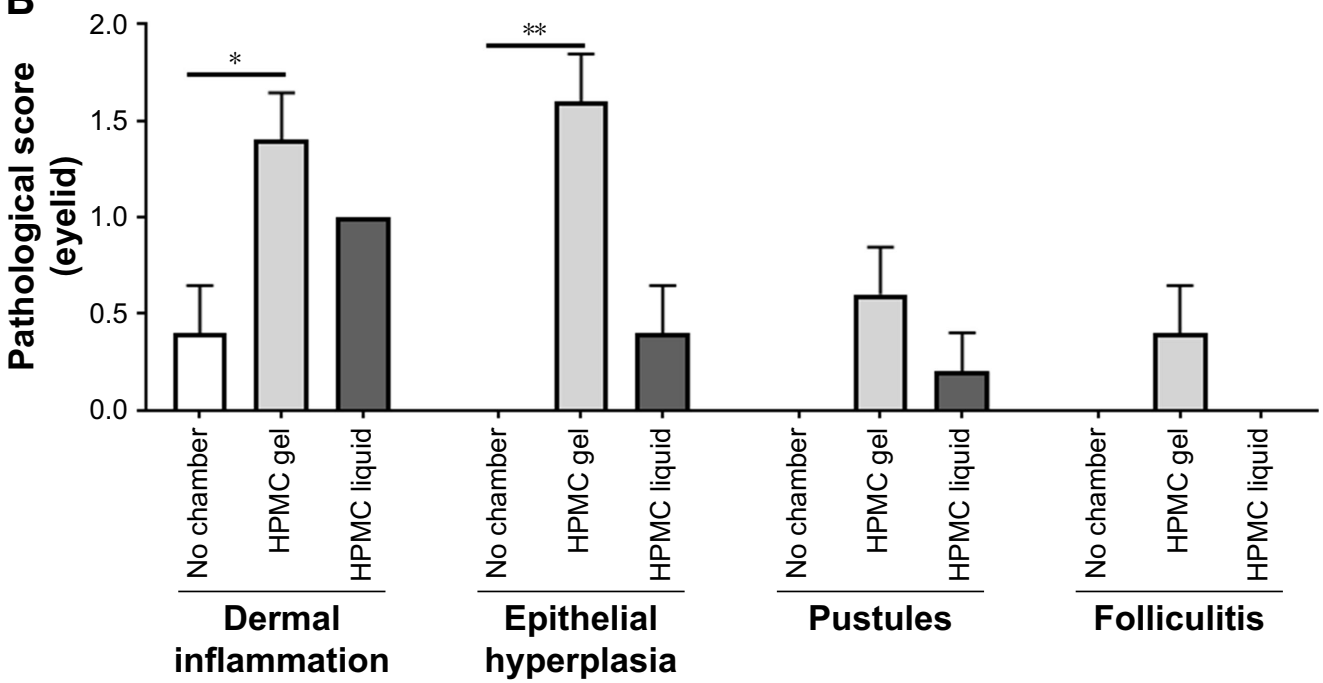

Figure 6 Histological analysis of guinea pig eyelid skin. Representative images of hematoxylin and eosin stained skin sections (A). Comparison of key pathology observations between treatment groups (B). Arrows indicate heterophilic inflammatory cells. Significant differences in dermal inflammation and epithelial hyperplasia were observed in scored eyelid sections between the HPMC gel treated groups and untreated controls. $* P<0.05 ; * * P<0.005$.

Abbreviation: HPMC, hydroxypropyl methylcellulose. 
feature of the OWC that allows for increased contact time of therapeutics on the ocular surface. This not only allows for the constant delivery of therapeutics, but also protects the eye when the eyelids are not capable of covering the surface of the eye. In addition to this, the constant therapeutic delivery that the watertight seal provides reduces the labor of ancillary hospital personnel by eliminating the need for manual drop instillation.

The results from this study demonstrate that existing PWD technology can be adapted for use on the injured ocular surface and uninjured periorbital tissues. Through the utilization of an in vivo guinea pig model of corneal epithelial wound healing, we found that corneal epithelial wounds in all treatment groups re-epithelialized by 72 hours post injury as determined by fluorescein uptake (Figure 1A). Furthermore, no clinically relevant corneal or dermal pathology was associated with short-term OWC use in this animal model. This suggests that it may be feasible to safely use the OWC on an injured ocular surface as a novel ophthalmic treatment option.

Initial studies performed by our lab using the OWC were aimed at testing this device on the uninjured eye (manuscript under review). As the corneal epithelium is crucial to the health of the cornea, this study was designed to assess how corneal re-epithelialization occurs in the fluid-filled environment provided by the OWC. When the corneal epithelium is absent, the ocular surface is compromised and fluid enters the cornea resulting in stromal edema. ${ }^{12}$ If the corneal epithelium has been debrided in its entirety, stromal edema can persist up to 6 weeks after the initial epithelial injury has healed, ${ }^{12}$ typically within $48-72$ hours post injury. In the current study, corneal thickness significantly increased at 24 hours after injury in all treatment groups as expected due to the corneal defect. After the epithelium was restored, around 48 hours, changes in corneal thickness were not significantly different from the uninjured, control corneas with the exception of those treated with HPMC liquid. The significant increase in corneal thickness persisted up to 72 hours post injury. While some corneal swelling is considered to be normal when an epithelial injury occurs, these results suggest that the properties of pharmaceuticals utilized in the OWC such as a liquid versus a gel could have varying effects on the cornea.

The OWC provides an environment that allows sustained delivery of therapeutics, while keeping the eye moisturized with the clinician's choice of solution or lubricant to include artificial tears. Artificial tears come in a variety of formulations including PVA, SH, CMC, HPMC (HMC), HP guar, and oil-based tears. The type of artificial tear used largely depends on the needs of the patient. When artificial tears or ophthalmic drops are utilized as a therapeutic, the drops quickly dissipate after administration. The ultimate goal is to correct the imbalance in the tear film, which is comprised of three layers: the mucin, aqueous, and lipid layer. Imbalances in any layer of the tear film can result in diseases such as dry eye or exposure keratopathy. ${ }^{22}$ When treating these patients, it may be important to consider the properties of the selected artificial tears and solutions delivered in the OWC as they may contribute to corneal swelling as observed in our previous studies utilizing BSS in the OWC (manuscript in preparation). When BSS was placed in the OWC, corneas demonstrated increased swelling when compared to those treated with HPMC. The results from this study and our previous studies indicate that a more viscous solution or gel such as HPMC may prove to be the most beneficial. Overall, while it appears to be safe to treat eyes with corneal epithelial defects with the OWC, these results indicate the importance of applying the most optimal solution and/or lubricant for use in the OWC.

Given that previous studies have investigated PWD technology and have shown that it allows for the application of increased concentrations and sustained delivery of antibiotics to the surface of the wound; ${ }^{17}$ it is conceivable that this technology would allow for a decrease in the sterilization time of the ocular surface, thereby decreasing the risk of infection and subsequent vision loss. Future studies will be expanded to determine if the adapted OWC technology can be employed successfully to treat injured periorbital tissue, prevent exposure keratopathy, and manage ocular infections.

\section{Conclusion}

The data collected in this study demonstrate that our OWC device is safe on sterile, corneal epithelial wounds when filled with HPMC gel or liquid. While not significantly different, our results also suggest that the vehicle utilized in the chamber in the presence of an epithelial defect be carefully considered as HPMC liquid-treated eyes appeared to heal more slowly. Furthermore, eyes treated with HPMC liquid showed significant corneal thickening compared to eyes treated with HPMC gel at 48 hours, but the difference between these two groups decreased and was no longer significant by 72 hours. Overall, these studies lend that the use of the OWC device is safe on the skin surrounding the eye and that OWC use does not affect corneal epithelial wound healing when HPMC gel is utilized. Further studies are needed to determine if this device can successfully treat other ocular disparities such as exposure keratopathy, ocular infections, and injured or burned periorbital tissues. 


\section{Abbreviations}

ANOVA, analysis of variance; BSS, balanced salt solution; CMC, carboxy methylcellulose; HP guar, hydroxypropyl guar; HPMC, hydroxypropyl methylcellulose; IACUC, Institutional Animal Care and Use Committee; IAF, Institute Armand Frappier; ICU, intensive care unit; IOP, intraocular pressure; NBF, neutral buffered formalin; OCT, optical coherence tomography; OWC, ocular wound chamber; PCED, persistent corneal epithelial defect; PVA, polyvinyl alcohol; PWD, platform wound device; SEM, standard error of mean; $\mathrm{SH}$, sodium hyaluronate.

\section{Acknowledgments}

The authors would like to gratefully acknowledge Mr André Akers for his assistance in obtaining professional photographic images and MAJ Nathan Wienandt for analysis of corneal and skin sections. This work was supported by the U.S. Army Medical Research and Material Command (MRMC) Clinical and Rehabilitative Medicine Research Program (PAD5; CRM0003). The opinions or assertions contained herein are the private views of the author and are not to be construed as official or as reflecting the views of the Department of the Army or the Department of Defense. Elof E Eriksson serves as a Chief Medical Officer at Applied Tissue Technologies. Dr Jennifer S McDaniel is an employee of Laulima Government Solutions, LLC. Dr Andrew W Holt, Dr Elaine D Por, Dr Anthony J Johnson, and Dr Gina L Griffith are employees of the U.S. government and this work was performed as part of their official duties.

\section{Disclosure}

The authors report no conflicts of interest in this work.

\section{References}

1. Bouchard CS, Morno K, Perkins J, McDonnell JF, Dicken R. Ocular complications of thermal injury: a 3-year retrospective. J Trauma. 2001;50(1):79-82.

2. Malhotra R, Sheikh I, Dheansa B. The management of eyelid burns. Surv Ophthalmol. 2009;54(3):356-371.

3. Grixti A, Sadri M, Edgar J, Datta AV. Common ocular surface disorders in patients in intensive care units. Ocul Surf. 2012;10(1):26-42.
4. Zhou Y, Liu J, Cui Y, Zhu H, Lu Z. Moisture chamber versus lubrication for corneal protection in critically ill patients: a meta-analysis. Cornea. 2014;33(11):1179-1185.

5. McCulley JP, Horowitz B, Husseini ZM, Horowitz M. Topical fibronectin therapy of persistent corneal epithelial defects. Fibronectin Study Group. Trans Am Ophthalmol Soc. 1993;91:367-386; discussion 386-390.

6. Lim P, Ridges R, Jacobs DS, Rosenthal P. Treatment of persistent corneal epithelial defect with overnight wear of a prosthetic device for the ocular surface. Am J Ophthalmol. 2013;156(6):1095-1101.

7. Katzman LR, Jeng BH. Management strategies for persistent epithelial defects of the cornea. Saudi J Ophthalmol. 2014;28(3):168-172.

8. Baum J. Thygeson lecture. Amniotic membrane transplantation: why is it effective? Cornea. 2002;21(4):339-341.

9. Griffith GL, Kasus-Jacobi A, Pereira HA. Bioactive antimicrobial peptides as therapeutics for corneal wounds and infections. Adv Wound Care (New Rochelle). 2017;6(6):175-190.

10. Perez-Merino P, Martinez-Garcia MC, Mar-Sardana S, et al. Corneal light transmission and roughness after refractive surgery. Optom Vis Sci. 2010;87(7):E469-E474.

11. Akpek EK, Gottsch JD. Immune defense at the ocular surface. Eye (Lond). 2003;17(8):949-956.

12. Carlson EC, Wang IJ, Liu CY, Brannan P, Kao CW, Kao WW. Altered KSPG expression by keratocytes following corneal injury. Mol Vis. 2003;9:615-623.

13. Yu FS, Yin J, Xu K, Huang J. Growth factors and corneal epithelial wound healing. Brain Res Bull. 2010;81(2-3):229-235.

14. Junker JP, Caterson EJ, Eriksson E. The microenvironment of wound healing. J Craniofac Surg. 2013;24(1):12-16

15. Breuing K, Andree C, Helo G, Slama J, Liu PY, Eriksson E. Growth factors in the repair of partial thickness porcine skin wounds. Plast Reconstr Surg. 1997;100(3):657-664.

16. Breuing K, Eriksson E, Liu P, Miller DR. Healing of partial thickness porcine skin wounds in a liquid environment. J Surg Res. 1992;52(1): 50-58.

17. Daly LT, Tsai DM, Singh M, et al. Topical minocycline effectively decontaminates and reduces inflammation in infected porcine wounds. Plast Reconstr Surg. 2016;138(5):856e-868e.

18. Junker JP, Lee CC, Samaan S, et al. Topical delivery of ultrahigh concentrations of gentamicin is highly effective in reducing bacterial levels in infected porcine full-thickness wounds. Plast Reconstr Surg. 2015; 135(1):151-159.

19. Griffith GL, Kasus-Jacobi A, Lerner MR, Pereira HA. Corneal wound healing, a newly identified function of CAP37, is mediated by protein kinase C delta (PKCdelta). Invest Ophthalmol Vis Sci. 2014;55(8): 4886-4895.

20. Leung VC, Belovay GW, Chan CC. Tegaderm(TM)dressing and Lacrilube ointment moisture chamber to manage exposure keratopathy. Can J Ophthalmol. 2016;51(5):e149-e151.

21. Hart DE, Simko M, Harris E. How to produce moisture chamber eyeglasses for the dry eye patient. J Am Optom Assoc. 1994;65(7): $517-522$.

22. Tseng SC, Tsubota $\mathrm{K}$. Important concepts for treating ocular surface and tear disorders. Am J Ophthalmol. 1997;124(6):825-835.
Clinical Ophthalmology

\section{Publish your work in this journal}

Clinical Ophthalmology is an international, peer-reviewed journal covering all subspecialties within ophthalmology. Key topics include: Optometry; Visual science; Pharmacology and drug therapy in eye diseases; Basic Sciences; Primary and Secondary eye care; Patien Safety and Quality of Care Improvements. This journal is indexed on

\section{Dovepress}

PubMed Central and CAS, and is the official journal of The Society of Clinical Ophthalmology (SCO). The manuscript management system is completely online and includes a very quick and fair peer-review system, which is all easy to use. Visit http://www.dovepress.com/ testimonials.php to read real quotes from published authors. 